Supplementary Information

\title{
Thermophoretic Detection of Exosomal microRNAs by
}

NanoFlares

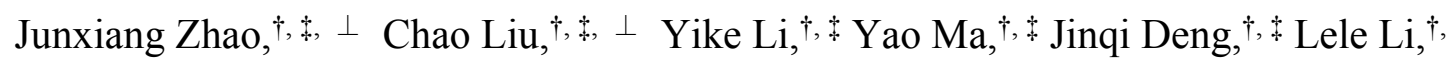

$\ddagger$ and Jiashu Sun $*, \dagger, \ddagger$

$\uparrow$ CAS Key Laboratory of Standardization and Measurement for Nanotechnology,

CAS Center for Excellence in Nanoscience, National Center for Nanoscience and

Technology, Beijing 100190, China.

$¥$ University of Chinese Academy of Sciences, Beijing 100049, China.

Email: sunjs@nanoctr.cn 


\section{Materials and Methods}

Microchamber fabrication. The microchamber was fabricated by sandwiching a $240-\mu \mathrm{m}$-thick spacer with a circular hole ( $7 \mathrm{~mm}$ in diameter) between a glass top layer (1 mm thick) and a sapphire bottom layer (1 mm thick).

Probe DNAs. The recognition sequences complementary to the target miRNAs were modified with thiol at $3^{\prime}$ end. The reporter DNA sequences were complementary to the corresponding recognition sequences with several bases shorter and modified with Cy5 at 5' end. All sequences used for the present study were summarized in Table S1. The synthesis was performed by Tsingke (Beijing, China).

Purity of the synthetic oligonucleotides. The purity of synthetic oligonucleotides (miR-375, miR-375 recognition sequence, and miR-375 reporter flare) was assessed by the combination of HPLC purification (LC3000U, CXTH, China) and subsequent linear ion trap mass spectrometric (LITMS) measurement (LTQ XL, Thermo scientific, USA). The spectrograms of synthetic miR-375, miR-375 recognition sequence, and miR-375 reporter flare showed sharp peaks at 6739, 9120, and $5211 \mathrm{Da}$, respectively (Figure S1). The relative differences between the peaked molecular weights and expected ones were only $0.05 \%, 0.03 \%$, and $0.09 \%$, respectively, indicating the high purity of synthetic oligonucleotides. ${ }^{\mathrm{S} 1}$ 
AuNP synthesis. $13 \mathrm{~nm}$ AuNPs were synthesized by citrate reduction of $\mathrm{HAuCl}_{4}$. All glass wares were washed by aqua regia $\left(\mathrm{HCl}: \mathrm{HNO}_{3}=3: 1\right)$, rinsed with deionized water, and dried in an oven. An aqueous solution of sodium citrate (6.8 $\mathrm{mM}, 50 \mathrm{~mL}$ ) was added into a three-necked flask with a condenser and heated to boiling for $5 \mathrm{~min}$. The solution was added with $\mathrm{HAuCl}_{4}(25 \mathrm{mM}, 0.5 \mathrm{~mL})$ quickly and boiled for another $15 \mathrm{~min}$. The solution was cooled at room temperature and incubated with $8 \mathrm{mg}$ of Bis(p-sulfonatophenyl)-phenylphosphine dipotassium salt (BSPP) overnight. ${ }^{\text {S2 }}$

NanoFlare fabrication. Recognition sequences $(100 \mu \mathrm{M}, 10 \mu \mathrm{L})$ and reporter flares $(100 \mu \mathrm{M}, 10 \mu \mathrm{L})$ were incubated at $37{ }^{\circ} \mathrm{C}$ for $45 \mathrm{~min}$ for hybridization. The hybridized duplexes were mixed with $10 \mathrm{nM}$ AuNPs to a final volume of $200 \mu \mathrm{L}$ and frozen at $-20{ }^{\circ} \mathrm{C}$ for 60 min to be modified onto the surfaces of AuNPs. The resulting NanoFlares were washed 3 times by ultrafiltration (Amicon, $100 \mathrm{kDa} 0.5$ $\mathrm{mL}$, Germany) at $10000 \times \mathrm{g}(10 \mathrm{~min}$ for each time $)$ and stored at $4{ }^{\circ} \mathrm{C}$ before use. The concentrations of NanoFlares were determined as $\sim 20 \mathrm{nM}$ by UV-Vis spectrometry on the basis of molar extinction coefficient of $2.7 \times 10^{8} \mathrm{M}^{-1} \mathrm{~cm}^{-1}$ at 524 nm. ${ }^{\mathrm{S} 3}$

Determination of the number of DNAs modified on each AuNP. We used excess glutathione (3.3 mM, GSH, Energy Chemical, China) to fully displace the hybridized duplexes from the functionalized AuNPs $(2.5 \mathrm{nM})$. The concentration of 
the displaced hybridized duplexes was determined as $113.7 \pm 5.4 \mathrm{nM}$ by comparing its fluorescence intensity against the calibration curve (Figure S2), indicating that 45 hybridized duplexes were modified on each AuNP. ${ }^{\mathrm{S} 4}$

Absorption spectra. The absorption spectra of AuNPs ( $5 \mathrm{nM})$, NanoFlares (miR, 5 $\mathrm{nM}$ ), and free DNA (the mixture of $225 \mathrm{nM}$ miR-375 recognition sequences and $225 \mathrm{nM}$ miR-375 reporter flares) were measured by a microplate reader (Synergy H1, Biotek, USA) with a scanning range of $300-700 \mathrm{~nm}$ and a step of $2 \mathrm{~nm}$.

NanoFlare characterization. After incubation of synthetic miR-375 targets (2 $\mu \mathrm{M})$ with NanoFlares ( $1 \mathrm{nM}, \sim 45$ recognition sequences per NanoFlare), a 7-fold enhancement in fluorescence was obtained by measuring the absorbance at $670 \mathrm{~nm}$ using a microplate reader (Synergy H1, Biotek, USA) (Figure 3a). This result suggested $\sim 88 \%$ fluorescence quenching efficiency for fluorophores by AuNPs, which was comparable to that of $75-95 \%$ in previous investigations. ${ }^{\mathrm{S} 5-6}$

Specificity in recognizing targeted miRNAs of NanoFlares. Orthogonal experiments were performed by mixing NanoFlares $(1 \mathrm{nM})$ targeting miR-375, miR-221, miR-210, and miR-10b with $2 \mu \mathrm{M}$ synthetic miR-375, miR-221, miR-210, and miR-10b at a sample volume of $100 \mu \mathrm{L}$. A solution of NanoFlares targeting miR-375 $(1 \mathrm{nM}, 100 \mu \mathrm{L})$ was used as a control sample. The fluorescence intensities of the samples were measured by a microplate reader (Biotek, USA) 
using an excitation wavelength of $630 \mathrm{~nm}$ and an emission wavelength of $670 \mathrm{~nm}$. The experiments were independently repeated by 3 times.

Cell lines. Human breast cancer cell lines MCF-7, MDA-MB-231, and SK-BR-3 were obtained from American Type Culture Collection (ATCC, USA). MCF-7 cells were cultured in RPMI-1640 medium (Gibco, USA). MDA-MB-231 cells were cultured in L-15 medium (Keynentec, China). SK-BR-3 cells were cultured in McCoy's5A medium (Keynentec, China). All media were supplemented with $10 \%$ EXOs-depleted fetal bovine serum (obtained by $12 \mathrm{~h}$ of ultracentrifugation at $150000 \times \mathrm{g}$ ) and $1 \%$ penicillin/streptomycin (Wisent, Toronto, Canada). All cell lines were incubated at $37{ }^{\circ} \mathrm{C}$ with $5 \% \mathrm{CO}_{2}$.

Clinical samples. The clinical serum samples were collected and processed following the Early Detection Research Network (EDRN) standard operating procedure (SOP): (1) blood samples from patients were collected into acid citrate dextrose (ACD) serum separator tubes (yellow top) and the tubes were gently inverted for $5-8$ times, (2) the tubes were stored upright at $4{ }^{\circ} \mathrm{C}$ for $30 \mathrm{~min}$, (3) the tubes were centrifuged at $2000 \times \mathrm{g}$ for $30 \mathrm{~min}$, (4) serum samples were pipetted out into labeled centrifuge tubes and stored at $-80{ }^{\circ} \mathrm{C}$ until measurement. Human sera were collected at the Chinese PLA General Hospital with informed consent. The patient status was summarized in Table S3. 
EXO isolation by differential centrifugation. To isolate EXOs derived from cell lines, the cell culture media were collected until cells reached a confluency of $70 \%$. The collected media $(300 \mathrm{~mL})$ were centrifuged first at $300 \times \mathrm{g}$ for $10 \mathrm{~min}$ and subsequently at $2000 \times \mathrm{g}$ for $10 \mathrm{~min}$ to remove cells and large debris. The supernatant was centrifuged at $10000 \times \mathrm{g}$ for $60 \mathrm{~min}$, processed by membrane filtration $(0.22 \mu \mathrm{m}$, Millipore, USA), and ultracentrifuged at $100000 \times \mathrm{g}$ for $3 \mathrm{~h}$. The EXOs were obtained by resuspending the pellet in $200 \mu \mathrm{L} 1 \times$ PBS. To isolate EXOs from clinical serum samples, serum was diluted by 100 folds in $1 \times$ PBS and centrifuged at $14000 \times \mathrm{g}$ for $20 \mathrm{~min}$ to remove cell debris. The supernatant was processed by membrane filtration $(0.22 \mu \mathrm{m}$, Millipore, USA), and subjected to twice of ultracentrifugation at $100000 \times \mathrm{g}(3 \mathrm{~h}$ for each time). The pellet was resuspended in $1 \times$ PBS as serum EXOs. Before ultracentrifugation, the medium was packaged into eight ultracentrifuge tubes with the weight difference between every pair of tubes smaller than $0.05 \mathrm{~g}$.

Dynamic light scattering (DLS). The size and surface zeta potential of NanoFlares were obtained by DLS measurements $(n=3)$ using a Malvern 3000HS Zetasizer (Malvern Instruments Ltd, England). The refractive indexes for the solvent and AuNP were set as 1.33 and 2.3, respectively.

Nanoparticle tracking analysis (NTA). To quantify the concentration and size distribution of EXOs, EXO samples were characterized using NTA (NanoSight 
NS500, Malvern Instrument, England) at $20 \pm 3{ }^{\circ} \mathrm{C}$. EXO concentrations were adjusted to $10^{8}-10^{9}$ particles $\mathrm{mL}^{-1}$ to achieve optimal counting accuracy. The data of size distribution were captured and analysed with the NTA 2.2 Analytical Software Suite.

Transmission electron microscopy (TEM). To observe the morphology of NanoFlares, $10 \mu \mathrm{L}$ aqueous solution of NanoFlares targeting miR-375 (NanoFlares (miR)) was absorbed on Formvar/carbon-coated copper grids. After air drying, the grids were observed on a Tecnai $\mathrm{G}^{2} 20$ S-TWIN TEM (FEI, USA) at $200 \mathrm{kV}$. To observe the internalization of NanoFlares into EXOs by Tecnai $G^{2} 20$ S-TWIN TEM, MCF-7 EXOs $\left(10^{10} \mathrm{~mL}^{-1}\right)$ were incubated with $0.2 \mathrm{nM}$ NanoFlares (miR) for $2 \mathrm{~h}$ at $37{ }^{\circ} \mathrm{C}$, fixed by $2 \%$ paraformaldehyde, and absorbed on Formvar/carbon-coated copper grids for $5 \mathrm{~min}$. Filter paper was used to blot extra EXO solution. Then EXOs were strained by 1 drop of $2 \%$ uranyl acetate, which was immediately blotted by filter paper after dropping on the grids. The staining step was repeated for 4 times. After air drying, the grids were observed on a Tecnai $\mathrm{G}^{2} 20$ S-TWIN TEM (FEI, USA) at $200 \mathrm{kV}$. A suspension MCF-7 EXOs $\left(10^{10}\right.$ $\mathrm{mL}^{-1}$ ) was used as control and observed following the same protocol.

Scanning transmission electron microscopy (STEM). To observe the uptake of NanoFlares by EXOs, MCF-7 EXOs $\left(10^{10} \mathrm{~mL}^{-1}\right)$ were incubated with $0.2 \mathrm{nM}$ NanoFlares (miR) for $2 \mathrm{~h}$, fixed by $2 \%$ paraformaldehyde, and absorbed on 
Formvar/carbon-coated copper grids for $30 \mathrm{~min}$. Filter paper was used to blot extra solution. Then EXOs were strained by 1 drop of $2 \%$ uranyl acetate, which was immediately blotted by filter paper after dropping on the grids. The staining step was repeated for 4 times. After air drying, the grids were observed on a SU8220 SEM (HITACHI, Japan). STEM bright field and dark field images were taken at 30 $\mathrm{kV}$.

Analysis of signal to noise (S/N) ratio. Under optimized experimental conditions, TSN exhibited a high $\mathrm{S} / \mathrm{N}$ ratio of 80 for detection of exosomal miRNA (Figure S10), because the size-dependent thermophoretic accumulation of EXOs (30 - 150 $\mathrm{nm})$ greatly enhanced the fluorescence signal of NanoFlares upon binding to miRNA targets in EXOs. Meanwhile, it was difficult to enrich free NanoFlares of small size $(\sim 18 \mathrm{~nm})$ by thermophoresis, and the background fluorescence of dispersed NanoFlares was relatively low under the fluorescence microscope.

Fluorescence co-localization. To validate the entry of NanoFlares into EXOs, MCF-7 EXOs $\left(10^{10} \mathrm{~mL}^{-1}\right)$ labeled by FAM-conjugated EpCAM aptamer $(0.25 \mu \mathrm{M})$ were incubated with unquenched NanoFlares $(0.2 \mathrm{nM})$ modified with recognition sequences distally conjugated with Cy5 at $37^{\circ} \mathrm{C}$ for $2 \mathrm{~h}$. The co-localization of FAM and Cy5 fluorescence was observed under a 100× objective on a fluorescent microscope (DMi8, Leica, Germany) equipped with a sCMOS camera (95B, Photometrics, Canada). The bright field images were also taken at the same scale, 
showing that the samples did not contain large debris or aggregates. The fluorescence co-localization events were counted from $\sim 20$ non-overlapping views. The percent of EXOs encapsulated with NanoFlares was determined as $22.5 \%$ on the basis of 57 events of co-localization of NanoFlares (Cy5) and EXOs (FAM) from 253 EXOs (FAM) (Figure S7 and Table S2). After $10 \mathrm{~min}$ laser irradiation $(1480 \mathrm{~nm})$, the percent of NanoFlare-loaded EXOs was remained as $23.8 \%$ on the basis of 43 co-localization events from 180 EXOs (FAM). Previous studies showed that most EXOs contained less than 1 copy of a given miRNA. ${ }^{\mathrm{S} 7}$ For example, the average abundance of miR-375 in MCF-7 EXOs was 0.01 copy per EXO. As long as one NanoFlare was internalized, its $\sim 45$ recognition sequences were sufficient for signaling the exosomal miRNA of low concentrations.

Experimental conditions. For evaluation of thermophoretic accumulation of EXOs, MCF-7 EXOs isolated by differential centrifugation were diluted to a concentration of $10^{10} \mathrm{~mL}^{-1}$ in $1 \times$ PBS and incubated with $0.2 \mathrm{nM}$ NanoFlares $(\mathrm{miR})$ at $37^{\circ} \mathrm{C}$ for $2 \mathrm{~h}$. For validation of the specific detection of miR-375 in EXOs, following control groups were used: (1) MCF-7 EXOs $\left(10^{10} \mathrm{~mL}^{-1}\right.$ in $\left.1 \times \mathrm{PBS}\right)$ incubated with $0.2 \mathrm{nM}$ NanoFlare (miR) and $170 \mathrm{pM}$ synthetic miR-375 at $37{ }^{\circ} \mathrm{C}$ for $2 \mathrm{~h}$; (2) MCF-7 EXOs $\left(10^{10} \mathrm{~mL}^{-1}\right.$ in $\left.1 \times \mathrm{PBS}\right)$ incubated with $0.2 \mathrm{nM}$ NanoFlare $(\mathrm{Rdm})$ at $37{ }^{\circ} \mathrm{C}$ for $2 \mathrm{~h}$; (3) $0.2 \mathrm{nM}$ NanoFlare (miR) incubated with $170 \mathrm{pM}$ synthetic miR-375 at $37{ }^{\circ} \mathrm{C}$ for $2 \mathrm{~h}$. To estimate the limit of detection and linear range, MCF-7 EXOs isolated by differential centrifugation were diluted in $1 \times \mathrm{PBS}$ 
to various concentrations of $10^{6}-10^{10} \mathrm{~mL}^{-1}$ and incubated with $0.2 \mathrm{nM}$ NanoFlares (miR) at $37{ }^{\circ} \mathrm{C}$ for $2 \mathrm{~h}$. For detecting exosomal miRNAs from clinical serum samples, aliquots of EXOs isolated from $0.5 \mu \mathrm{L}$ serum were diluted in $1 \times \mathrm{PBS}$ and incubated with $0.2 \mathrm{nM}$ NanoFlares targeting miR-375, miR-221, miR-210, and miR-10b, and NanoFlares $(\mathrm{Rdm})$ at $37{ }^{\circ} \mathrm{C}$ for $2 \mathrm{~h}$. The incubated samples were fixed by $2 \%$ paraformaldehyde at room temperature for $30 \mathrm{~min}$, stopped using 50 $\mathrm{mM}$ glycine, and adjusted to a total volume of $10 \mu \mathrm{L}$, corresponding to 20 fold dilution of initial serum. For thermophoretic assay, $10 \mu \mathrm{L}$ of incubated samples were filled in a $240 \mu \mathrm{m}$ high microchamber that mounted on an inverted fluorescence microscope (Nikon, Japan). An infrared laser $(170 \mathrm{~mW}, 1480 \mathrm{~nm}$, Changchun Laser Optoelectronics Technology, China) was focused on the microchamber bottom using a $40 \times$ objective (N.A. 0.22 ) to generate temperature gradient for $10 \mathrm{~min}$. The fluorescence images before and after laser irradiation were observed under a $40 \times$ objective and recorded by an electron multiplying CCD (EMCCD, Andor, England) with a $2 \times 2$ pixel binning and an exposure time of 50 ms.

Optimization of detection conditions. To evaluate whether the fixation can improve the detection performance, $0.2 \mathrm{nM}$ NanoFlares (miR) were incubated with $2 \%$ paraformaldehyde at room temperature for $30 \mathrm{~min}$ and stopped using $50 \mathrm{mM}$ glycine. $0.2 \mathrm{nM}$ NanoFlare (miR) suspension without paraformaldehyde fixation was used as control. The two samples were subjected to thermophoretic 
accumulation using a $1480 \mathrm{~nm}$ laser irradiation for $10 \mathrm{~min}$. The fluorescence intensity of fixed NanoFlares was 14 times lower than that of control, indicating significant reduction of background signal through fixation.

To optimize the laser irradiation time, MCF-7 EXOs were diluted to a concentration of $10^{10} \mathrm{~mL}^{-1}$ in $1 \times \mathrm{PBS}$, incubated with $0.2 \mathrm{nM}$ NanoFlares (miR) and $2 \mathrm{mM} \mathrm{MgCl} 2$ at $37{ }^{\circ} \mathrm{C}$ for $2 \mathrm{~h}$, fixed using $2 \%$ paraformaldehyde for $30 \mathrm{~min}$, stopped by $50 \mathrm{mM}$ glycine, and subjected to laser heating up to $20 \mathrm{~min}$. The fluorescence intensity reached the maximum at $10 \mathrm{~min}$ and did not further increase with longer laser irradiation time.

For optimization of NanoFlare concentration, MCF-7 EXOs were diluted to a concentration of $10^{10} \mathrm{~mL}^{-1}$ in $1 \times \mathrm{PBS}$, incubated with $2 \mathrm{mM} \mathrm{MgCl} 2$ and NanoFlares (miR) of different concentrations $(0.1,0.2$, and $0.5 \mathrm{nM})$ at $37^{\circ} \mathrm{C}$ for $2 \mathrm{~h}$, fixed using $2 \%$ paraformaldehyd for $30 \mathrm{~min}$, stopped by $50 \mathrm{mM}$ glycine, and subjected to laser heating for $10 \mathrm{~min}$. The optimal concentration of NanoFlares was determined to be $0.2 \mathrm{nM}$ on the basis of a maximum $\mathrm{S} / \mathrm{N}$ ratio of 80 (Figure $\mathrm{S} 9$ ).

For optimization of dilution fold of clinical serum sample, EXOs isolated from serum of 1 breast cancer (BC) patient and 1 healthy donor (HD) were diluted by 20, 50, and 100 folds. The fluorescence intensity ratio of $\mathrm{BC}$ to $\mathrm{HD}$ monotonically increased from 2 to 3 with the decrease in dilution fold.

For optimization of incubation time, serum EXOs from a BC patient (20-fold dilution) were incubated with $2 \mathrm{mM} \mathrm{MgCl}_{2}$ and $0.2 \mathrm{nM}$ NanoFlares (miR) at $37{ }^{\circ} \mathrm{C}$ for different time periods up to $3 \mathrm{~h}$ before laser heating. The fluorescence intensity 
gradually increased with incubation time up to $2 \mathrm{~h}$ and did not further increase with longer incubation time (Figure S8).

Exosomal miRNA detection using molecular beacons (MBs). We used a molecular beacon (MB-375) to detect miR-375 in MCF-7 EXOs by thermophoretic accumulation. $^{\mathrm{S} 8}$ MB-375 is a small stem-loop oligonucleotide probe with a fluorophore and quencher. The sequence of MB-375 was 5'-Cy5-CGC GAT CTC ACG CGA GCC GAA CGA ACA AAG ATC GCG -BHQ3-3' (Table S1). The middle 22 nucleotides within the loop region were complementary to miR-375. To induce the uptake of MB-375 by EXOs, $0.9 \mu \mathrm{L}$ MB-375 $\left(10^{5} \mathrm{nM}\right)$ diluted in 49.1 $\mu \mathrm{L}$ opti-MEM culture medium was mixed with $50 \mu \mathrm{L}$ transfection agent Lipofectamine 2000 (Life Technologies, USA) and incubated at room temperature for $10 \mathrm{~min}$ to form Lipofectamine 2000/MB-375 (Lipo/MB) complexes. For thermophoretic detection of miR-375 in MCF-7 EXOs, 89.1 $\mu \mathrm{L}$ MCF-7 EXOs prepared at different concentrations ranging from $10^{7}$ to $10^{10} \mathrm{~mL}^{-1}$ were mixed with $0.9 \mu \mathrm{L}$ Lipo/MB complexes (the final concentration of MB-375 was $9 \mathrm{nM}$, equivalent to that of recognition probes of NanoFlares), incubated at $37^{\circ} \mathrm{C}$ for $2 \mathrm{~h}$, and subjected to thermophoretic signal amplification for $10 \mathrm{~min}$. Lipo/MB complexes displayed a LoD of $18 \mathrm{fM}$ for thermophoretic detection of miR-375 in MCF-7 EXOs, which was 50 folds higher than that of NanoFlares (0.36 fM, Figure S16). 
Polymerase chain reaction (PCR). Total RNA was extracted from the EXOs derived from MCF-7, MDA-MB-231, and SK-BR-3 cells by using miRcute Serum/Plasma miRNA Isolation Kit (TIANGEN Biotech, China). The quantity and quality of the extracted RNA were measured by NanoDrop (Agilent Technologies, USA) and stored at $-80{ }^{\circ} \mathrm{C}$ until use. Complementary DNAs (cDNAs) were synthesized by using miRcute Plus miRNA First-Strand cDNA Kit (TIANGEN Biotech, China). $70 \mathrm{ng}$ of total RNA ( $20 \mu \mathrm{L}$ in volume) was added with poly(A) tailing reaction mixture, followed by polyadenylation and reverse transcription reaction at $42{ }^{\circ} \mathrm{C}$ for $60 \mathrm{~min}$ and reaction stop at $95{ }^{\circ} \mathrm{C}$ for $3 \mathrm{~min}$. The cDNA product was diluted by 10 folds and $2 \mu \mathrm{L}$ of diluted cDNA was mixed with $10 \mu \mathrm{L}$ of $2 \times$ miRcute Plus miRNA PreMix (TIANGEN Biotech, China), $0.4 \mu \mathrm{L}$ of forward and reverse primers (10 $\mathrm{nM}$ ), has-miR-U6 (as housekeeping gene), and RNase-free water to a final volume of $20 \mu \mathrm{L}$. The reaction was performed and monitored by a real-time PCR detection system (Applied Biosystem, 7500) using the following thermocycler conditions: pre-denature at $95{ }^{\circ} \mathrm{C}$ for $15 \mathrm{~min}$ and 40 cycles of denature $\left(94{ }^{\circ} \mathrm{C}\right.$ for 40 s) and anneal $\left(60^{\circ} \mathrm{C}\right.$ for $\left.34 \mathrm{~s}\right)$. For absolute quantification of miR-375 in MCF-7 EXOs, a standard curve was established using synthetic miR-375 with different concentrations ranging from $100 \mathrm{fM}$ to $1 \mathrm{nM}$. The recovery rate of miRNA after series procedures of RNA extraction, cDNA synthesis, and RT-PCR was determined as $35.6 \%$ by spiking Caenorhabditis elegans miRNA into EXO suspension prior to RNA extraction. Finally, the amount of miR-375 enclosed in 4.6 $\times 10^{10}$ MCF-7 EXOs was determined as $4.5 \times 10^{8}$ copies, indicating only $\sim 0.01$ 
miR-375 loaded per MCF-7 EXO.

Linear discrimination analysis (LDA). When discriminating $\mathrm{ER}+\mathrm{BC}$ and $\mathrm{HD}$ using combinations of multiple miRNA biomarkers, LDA algorithm was used to project high-dimensional signature $(\mathbf{x})$, i.e., the expression levels of the miRNAs onto a one-dimensional space. An optimal set of weighting factors (the best projection orientation, $\mathbf{W}_{\mathrm{T}}$ ) for $\mathbf{x}$ was automatically generated by LDA to maximize the discrimination power. The weighted $\operatorname{sum}\left(\mathbf{W}_{\mathrm{T}} \cdot \mathbf{x}\right)$ was transformed to the probability (LDA score, ranging from 0 to 1 ) of assigning a serum sample to ER+ $\mathrm{BC}$ or HD. LDA was performed with the linear algorithm using Matlab (Mathwork, USA).

Statistical analysis. The significance of difference in the expression levels from two different EXO samples was tested using a two-tailed $t$-test. To evaluate the performance of $\mathrm{ER}+\mathrm{BC}$ detection by individual miRNA markers, receiver operating characteristic (ROC) analysis was carried out. The LDA scores were used to evaluate the performance of different combinations of miRNA markers. Significance analyses, construction of ROC curve, and calculation of area under curve (AUC) were performed using GraphPad Prism 7 (GraphPad, USA). 
Table S1. Summary of sequences

\begin{tabular}{cc}
\hline Sequence name & Sequence (5'-3') \\
\hline Recognition-375 & TCACGCGAGCCGAACGAACAAAAAAAAAA-SH \\
Report flare-375 & Cy5-TTTGTTCGTTCGGCT \\
miR-375 & TTTGTTCGTTCGGCTCGCGTGA \\
Recognition-221 & GAAACCCAGCAGACAATGTAGCTAAAAAAA-SH \\
Report flare -221 & Cy5-AGCTACATTGTCTGC \\
miR-221 & AGCTACATTGTCTGCTGGGTTTC \\
Recognition-210 & CCAGCCGCTGTCACACGCACAGAAAAAAA-SH \\
Report flare -210 & CTGTGCGTGTGACAGCGGCTGA \\
miR-210 & Cy5-TACCCTGTAGAACCG \\
Recognition-10b & CACAAATTCGGTTCTACAGGGTAAAAAAAA-SH \\
Report flare -10b & TACCTGTAGAACCGAATTTGTG \\
miR10b & Cy5-TCACGCGAGCCGAACGAACAAAAAAAAAA-SH \\
Cy5-Recognition-375 & CyCGATCTCACGCGAGCCGAACGAACAAAGATCGCG-B \\
MB-375 &
\end{tabular}


Table S2. Summary of co-localization of NanoFlares (Cy5) and EXOs (FAM) before and after laser irradiation

\begin{tabular}{lcccc}
\hline \multicolumn{1}{c}{ Sample } & $\begin{array}{c}\text { NanoFlare } \\
\text { (Cy5) }\end{array}$ & $\begin{array}{c}\text { EXO } \\
\text { (FAM) }\end{array}$ & Co-localization & $\begin{array}{c}\text { Percent } \\
\text { (co-localization/EXO) }\end{array}$ \\
\hline Before laser & 1109 & 253 & 57 & $22.5 \%$ \\
After laser & 1029 & 180 & 43 & $23.8 \%$ \\
\hline
\end{tabular}


Table S3. Summary of breast cancer patients (BC) and healthy donors (HD)

\begin{tabular}{|c|c|c|c|}
\hline Index & Age & Stage & Subtype \\
\hline $\mathrm{BC} 1$ & 55 & I & ER+ HER2- \\
\hline $\mathrm{BC} 2$ & 62 & II & ER+ HER2- \\
\hline $\mathrm{BC} 3$ & 40 & II & ER+ HER2- \\
\hline $\mathrm{BC} 4$ & 52 & II & ER+ HER2- \\
\hline BC 5 & 32 & II & ER+ HER2- \\
\hline BC 6 & 33 & IV & ER+ HER2- \\
\hline $\mathrm{BC} 7$ & 52 & IV & ER+ HER2- \\
\hline BC 8 & 56 & IV & ER+ HER2- \\
\hline $\mathrm{BC} 9$ & 38 & IV & ER+ HER2- \\
\hline BC 10 & 50 & IV & ER+ HER2- \\
\hline BC 11 & 30 & II & ER+ HER2+ \\
\hline BC 12 & 54 & II & ER + HER2+ \\
\hline BC 13 & 62 & II & ER + HER2+ \\
\hline BC 14 & 56 & III & ER+ HER2+ \\
\hline BC 15 & 56 & IV & ER+ HER2+ \\
\hline BC 16 & 42 & IV & ER+ HER2+ \\
\hline BC 17 & 50 & IV & ER + HER2+ \\
\hline HD 1 & 35 & - & - \\
\hline HD 2 & 57 & - & - \\
\hline HD 3 & 39 & - & - \\
\hline HD 4 & 55 & - & - \\
\hline HD 5 & 48 & - & - \\
\hline HD 6 & 55 & - & - \\
\hline HD 7 & 55 & - & - \\
\hline HD 8 & 75 & - & - \\
\hline HD 9 & 48 & - & - \\
\hline HD 10 & 74 & - & - \\
\hline HD 11 & 43 & - & - \\
\hline HD 12 & 41 & - & - \\
\hline
\end{tabular}


Table S4. Performance of exosomal miRNAs in ER+ BC versus HD discrimination

\begin{tabular}{|c|c|c|c|c|}
\hline Marker panel & $\begin{array}{c}\text { Sensitivity } \\
(\%)\end{array}$ & $\begin{array}{c}\text { Specificity } \\
(\%)\end{array}$ & $\begin{array}{c}\text { Accuracy } \\
(\%) \\
\end{array}$ & $\mathbf{A U C}$ \\
\hline miR-375 & 94.1 & 83.3 & 89.7 & 0.96 \\
\hline $\operatorname{miR}-221$ & 64.7 & 75.0 & 69.0 & 0.81 \\
\hline miR-210 & 70.6 & 41.7 & 58.6 & 0.65 \\
\hline miR-10b & 64.7 & 58.3 & 62.1 & 0.69 \\
\hline $\operatorname{miR}-375+\operatorname{miR}-221$ & 70.6 & 91.1 & 79.3 & 0.83 \\
\hline $\operatorname{miR}-375+\operatorname{miR}-210$ & 88.2 & 83.3 & 86.2 & 0.90 \\
\hline miR-375 + miR-10b & 64.7 & 91.7 & 75.9 & 0.83 \\
\hline $\begin{array}{c}\mathrm{miR}-375+\mathrm{miR}-221+ \\
\mathrm{miR}-210\end{array}$ & 70.6 & 83.3 & 75.9 & 0.85 \\
\hline $\begin{array}{c}\mathrm{miR}-375+\operatorname{miR}-221+ \\
\mathrm{miR}-10 \mathrm{~b}\end{array}$ & 58.8 & 83.3 & 69.0 & 0.79 \\
\hline $\begin{array}{c}\mathrm{miR}-375+\mathrm{miR}-210+ \\
\mathrm{miR}-10 \mathrm{~b}\end{array}$ & 70.6 & 91.7 & 79.3 & 0.84 \\
\hline $\begin{array}{c}\operatorname{miR}-375+\operatorname{miR}-221+ \\
\operatorname{miR}-210+\operatorname{miR}-10 b\end{array}$ & 58.8 & 91.7 & 72.4 & 0.82 \\
\hline
\end{tabular}




\section{Supplementary Figures}
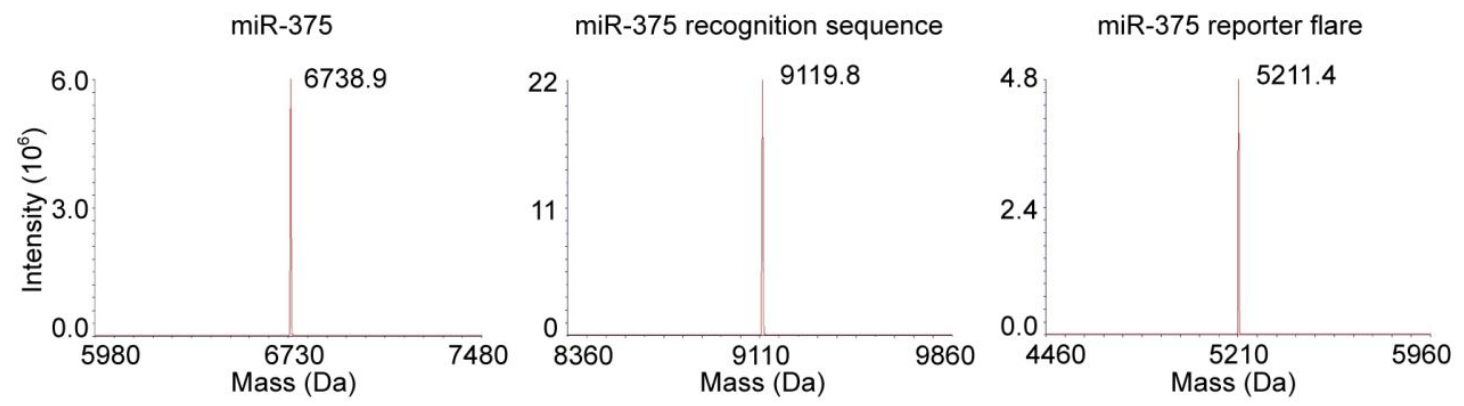

Figure S1. Characterization of synthetic miR-375, miR-375 recognition sequence, and miR-375 reporter flare by HPLC purification and MS measurement. 


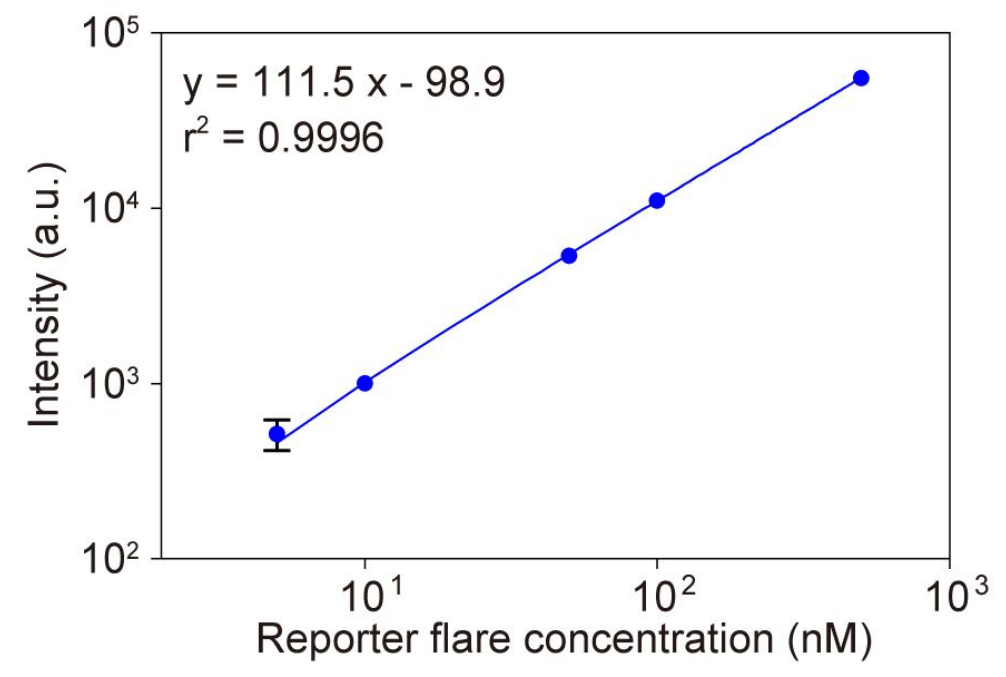

Figure S2. Standard curve for determining the number of Cy5-conjugated reporter flares on each NanoFlare. The fluorescence intensities of Cy5-conjugated reporter flares with different concentrations were measured by a microplate reader $(n=3$, mean \pm s.d.). 

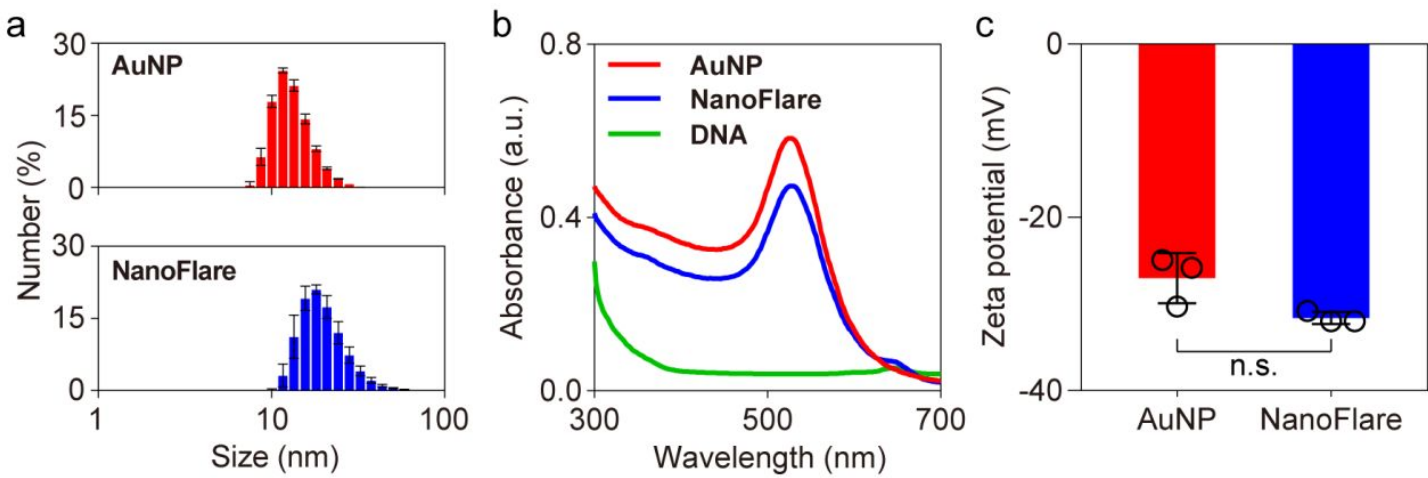

Figure S3. Characterization of NanoFlares. (a) DLS measurement of hydrodynamic sizes of AuNPs and NanoFlares (miR) ( $n=3$, mean \pm s.d.). (b) UV/Vis absorption spectra of the AuNPs ( $5 \mathrm{nM}$ ), NanoFlares (miR, $5 \mathrm{nM}$ ), and free DNA (the mixture of $225 \mathrm{nM}$ miR-375 recognition sequences and $225 \mathrm{nM}$ miR-375 reporter flares). (c) Zeta potential of the AuNPs and NanoFlares (miR) dispersed in DI water at $25^{\circ} \mathrm{C}$. NanoFlares were slightly more negative than AuNPs, which might be attributed to the functionalization of negatively charged DNA. ${ }^{\mathrm{S} 3}$ n.s. indicated no significance. 


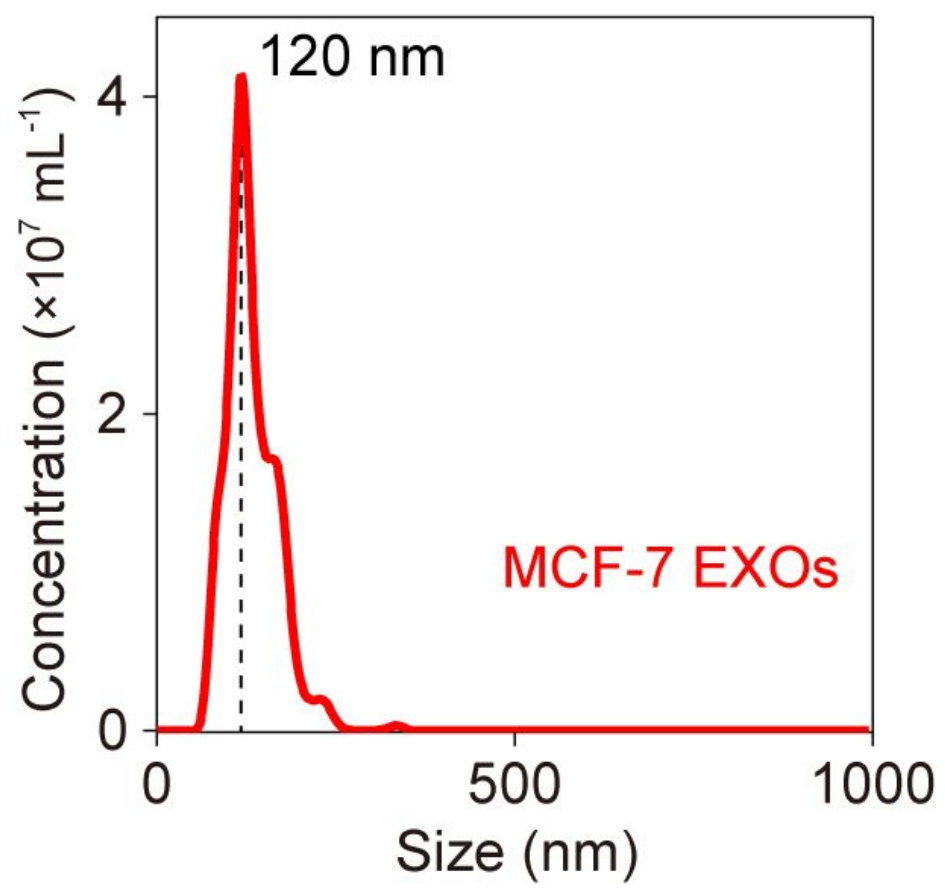

Figure S4. Size distribution of MCF-7 EXOs measured by NTA. Size mode, $120 \mathrm{~nm}$. 

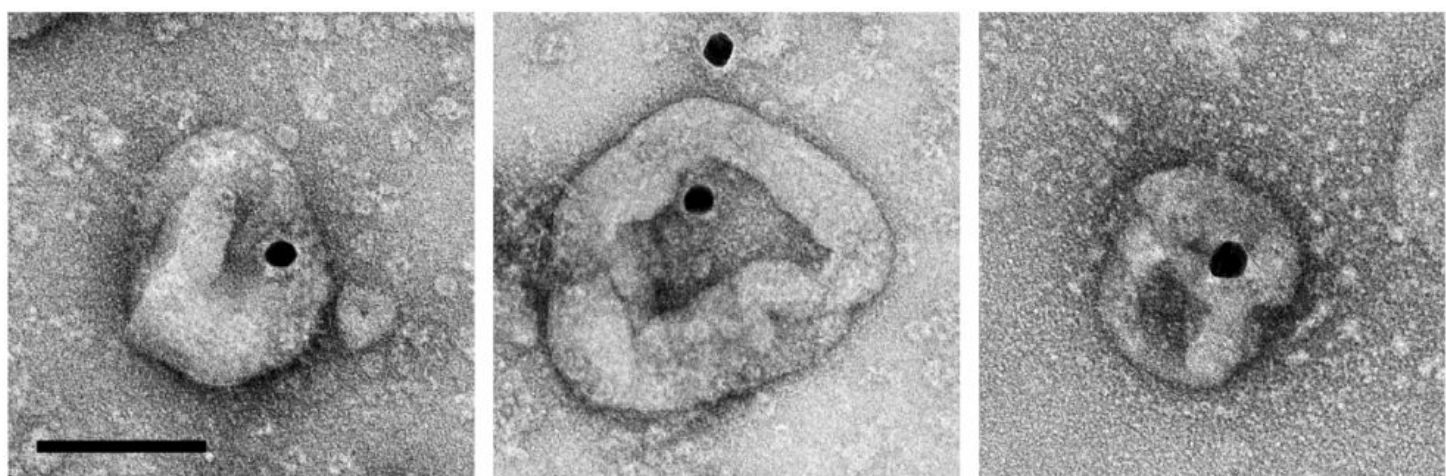

Figure S5. TEM images showing internalization of NanoFlares into EXOs after incubation for $2 \mathrm{~h}$. Scale bars, $100 \mathrm{~nm}$. 


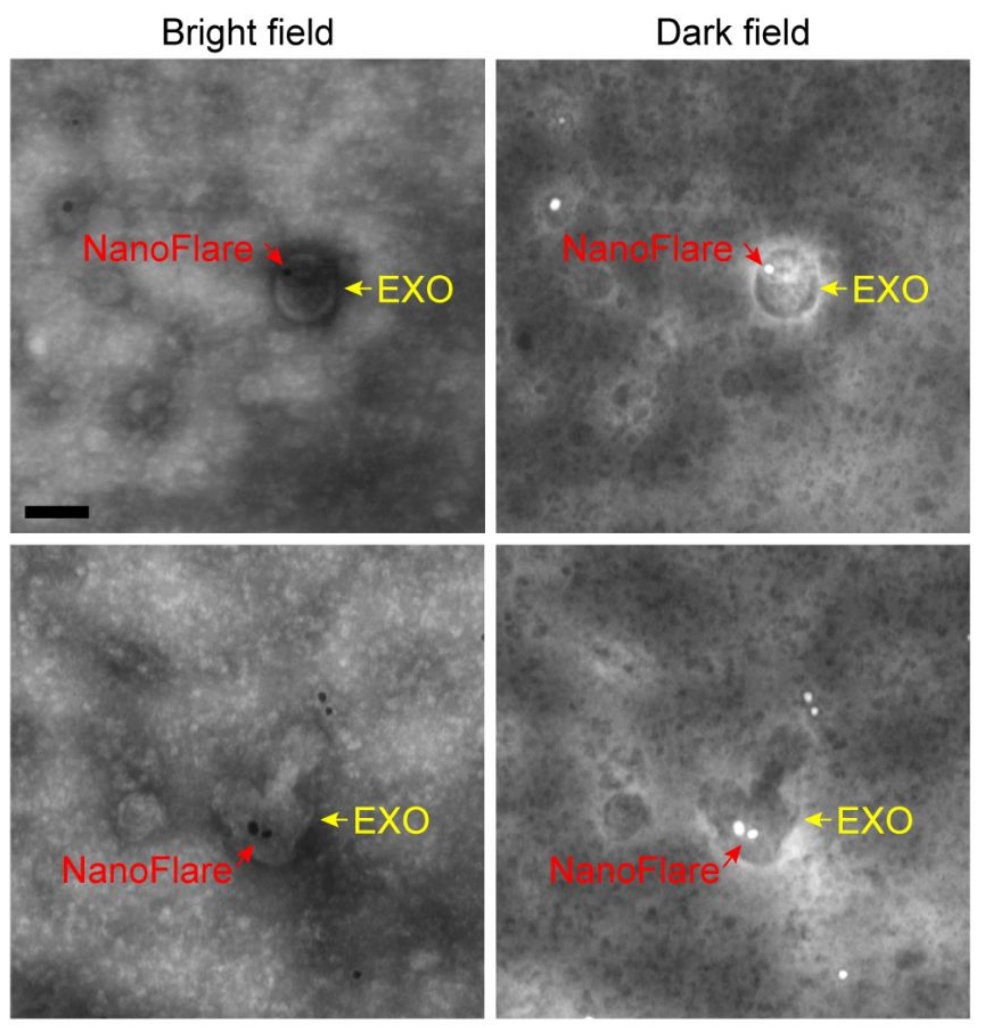

Figure S6. STEM bright field and dark field images of EXOs encapsulated with NanoFlares after incubation for $2 \mathrm{~h}$. Scale bar, $100 \mathrm{~nm}$. Yellow arrows indicated EXOs, and red arrows indicated NanoFlares. 


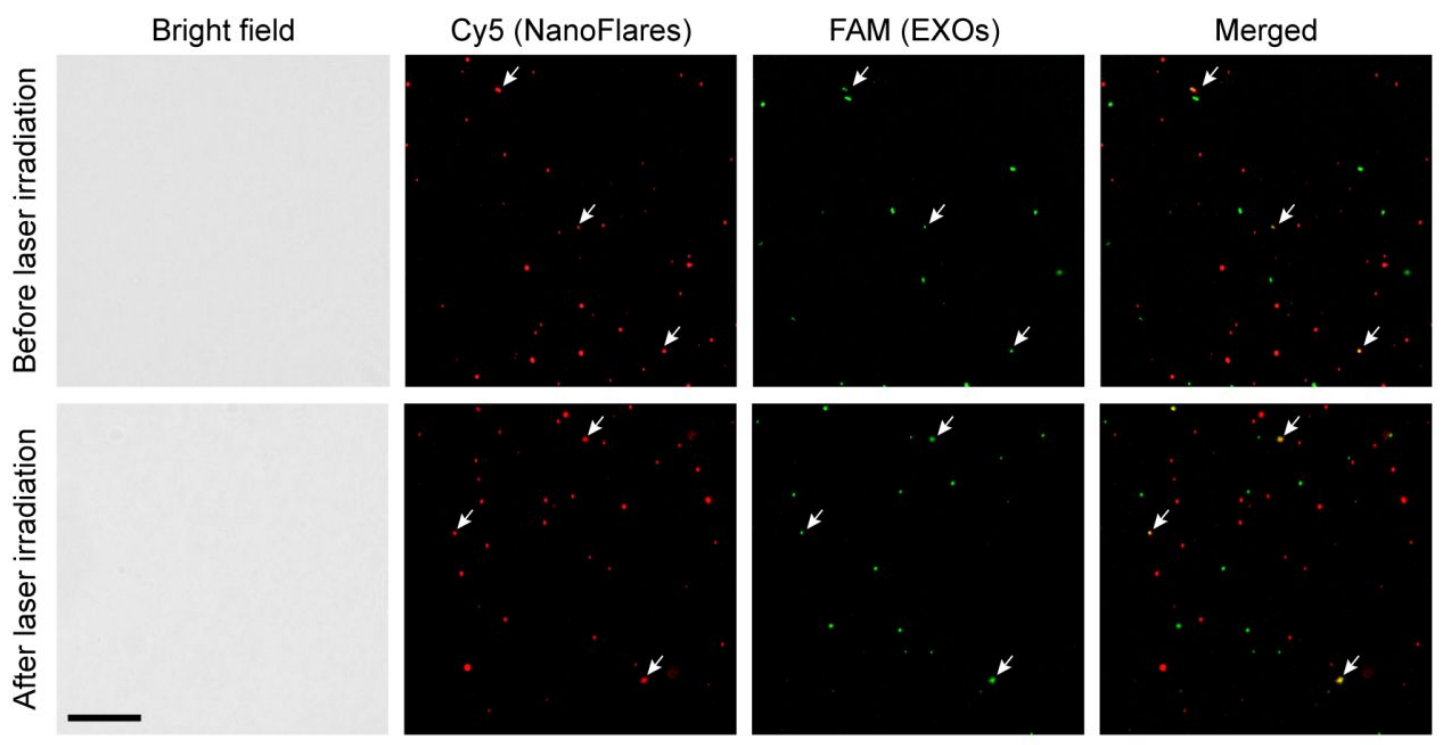

Figure S7. Fluorescence microscopy imaging of co-localization of Cy5 fluorescence from NanoFlares and FAM fluorescence from EXOs after incubation for $2 \mathrm{~h}$. Scale bar, $20 \mu \mathrm{m}$. White arrows indicated the successful co-localization of NanoFlares and EXOs. 


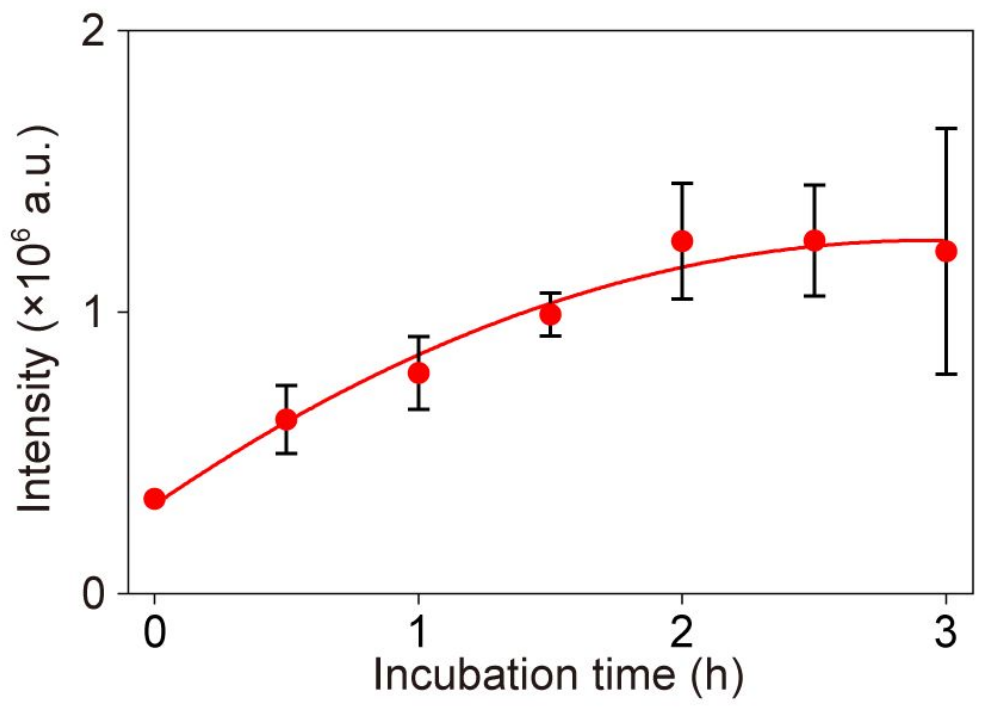

Figure S8. Optimization of incubation time. Fluorescence intensities measured at various incubation times ranging from 0 to $3 \mathrm{~h}$ with a NanoFlare concentration of 0.2 $\mathrm{nM}(n=3$, mean \pm s.d. $)$ 
a

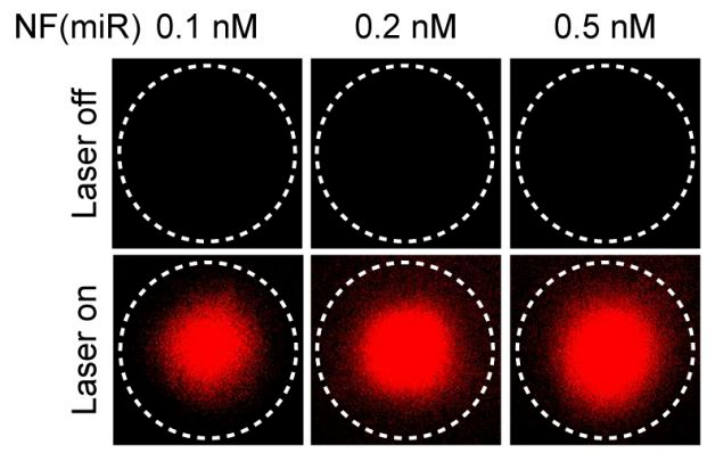

C

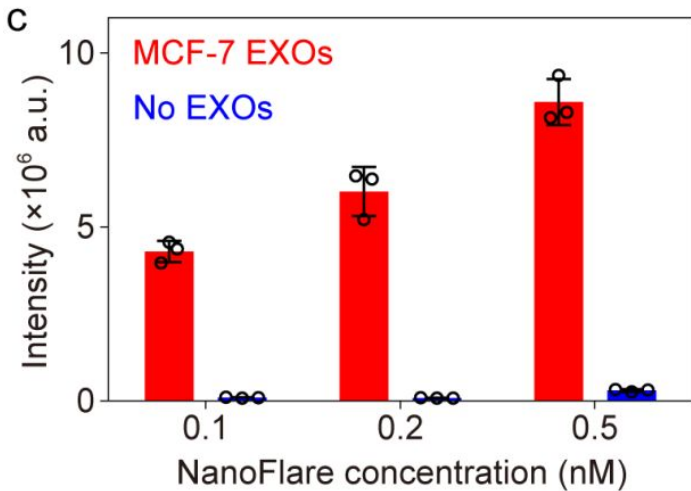

b

NanoFlares
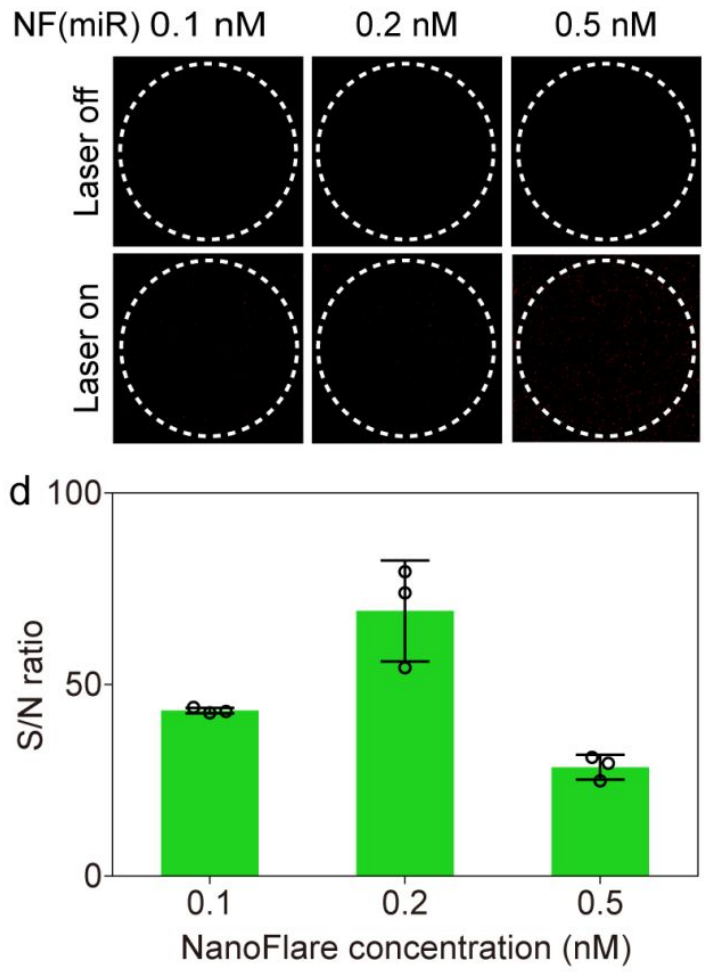

Figure S9. Optimization of NanoFlare concentration. (a) Fluorescence images of MCF-7 EXOs $\left(10^{10} \mathrm{~mL}^{-1}\right)$ treated with NanoFlares of different concentrations $(0.1$ to $0.5 \mathrm{nM}$ ) for detection of exosomal miR-375 by TSN. (b) Fluorescence images of NanoFlares of different concentrations $(0.1$ to $0.5 \mathrm{nM})$ subjected to laser irradiation. (c) Fluorescence intensities of MCF-7 EXOs encapsulated with NanoFlares and background noises of NanoFlares after laser irradiation $(n=3$, mean \pm s.d.). (d) $\mathrm{S} / \mathrm{N}$ ratio at different NanoFlare concentrations ( $n=3$, mean \pm s.d.). 


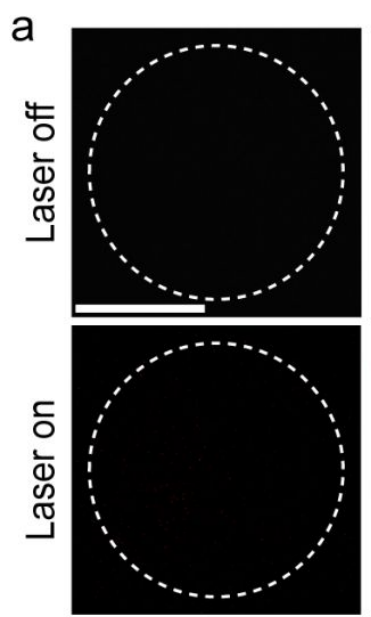

NanoFlares
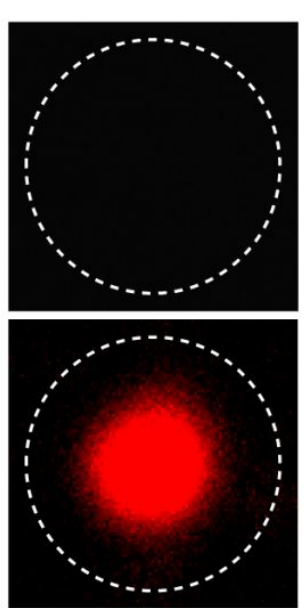

MCF-7 EXOs

+ NanoFlares

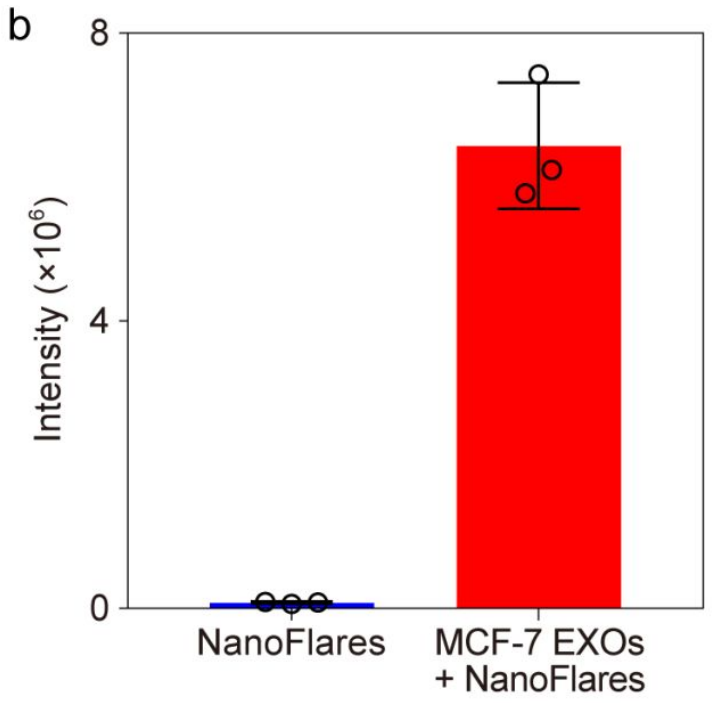

Figure S10. Analysis of signal to noise ratio ( $\mathrm{S} / \mathrm{N}$ ratio) of TSN. (a) Fluorescence images of NanoFlares $(0.2 \mathrm{nM})$ and MCF-7 EXOs $\left(10^{10} \mathrm{~mL}^{-1}\right)$ treated with NanoFlares (0.2 nM) after thermophoretic accumulation. Scale bar, $50 \mu \mathrm{m}$. (b) A high $\mathrm{S} / \mathrm{N}$ ratio of 80 was obtained. 

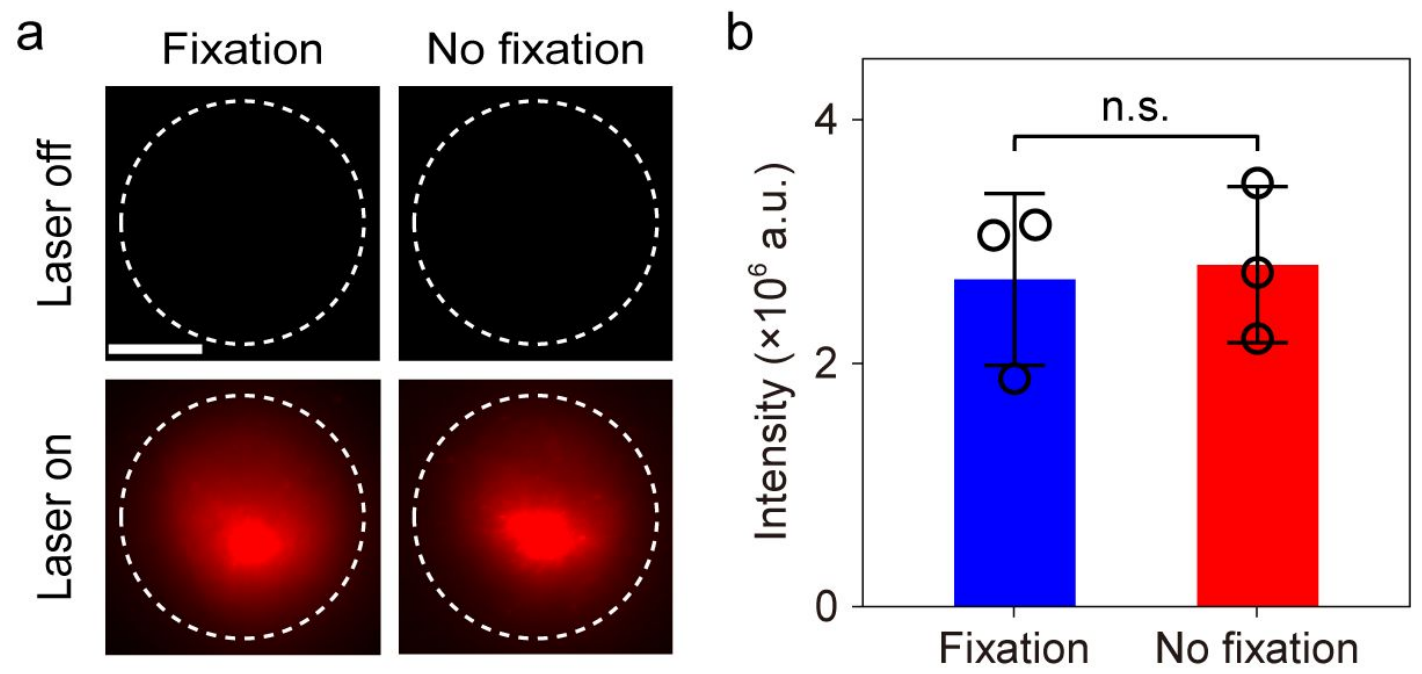

Figure S11. Negligible effect of fixation on thermophoretic focusing. (a) Fluorescence images and (b) fluorescence intensities of thermophoretic accumulation of $100 \mathrm{~nm}$ PS beads with or without adding $2 \%$ paraformaldehyde showed the same extent of accumulation. Scale bar, $50 \mu \mathrm{m}$. n.s. indicated no significance. 

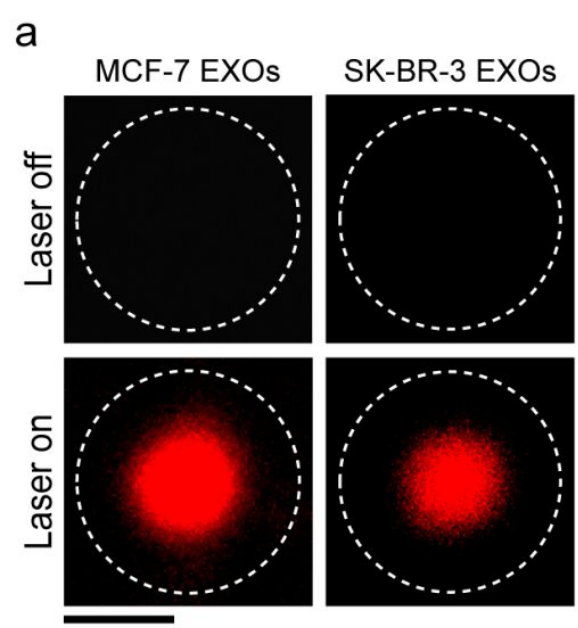

SK-BR-3 EXOs

$+\operatorname{miR}-375$

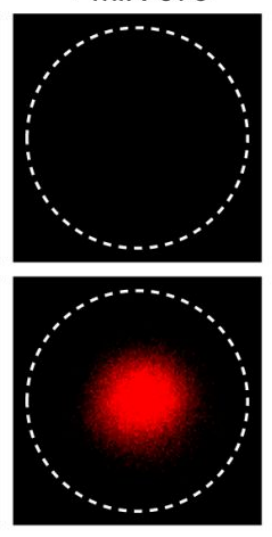

b

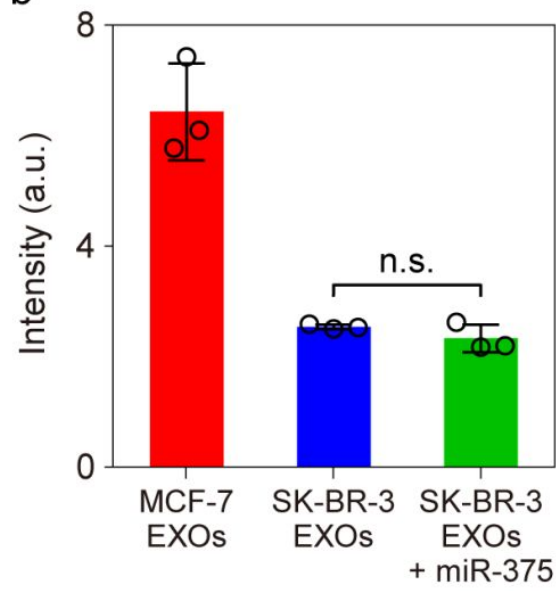

Figure S12. Detection of exosomal miR-375 in SK-BR-3 EXOs with or without adding synthetic miR-375 by TSN. (a) Fluorescence images and (b) fluorescence intensities of SK-BR-3 EXOs $\left(10^{10} \mathrm{~mL}^{-1}\right)$ treated with NanoFlares and the mixture of SK-BR-3 EXOs $\left(10^{10} \mathrm{~mL}^{-1}\right)$ and synthetic miR-375 targets $(170 \mathrm{pM})$ treated with NanoFlares after thermophoretic accumulation. The adding of miR-375 targets did not result in an increase in fluorescence intensity as compared to that of EXOs. Scale bar, $50 \mu \mathrm{m}$. 


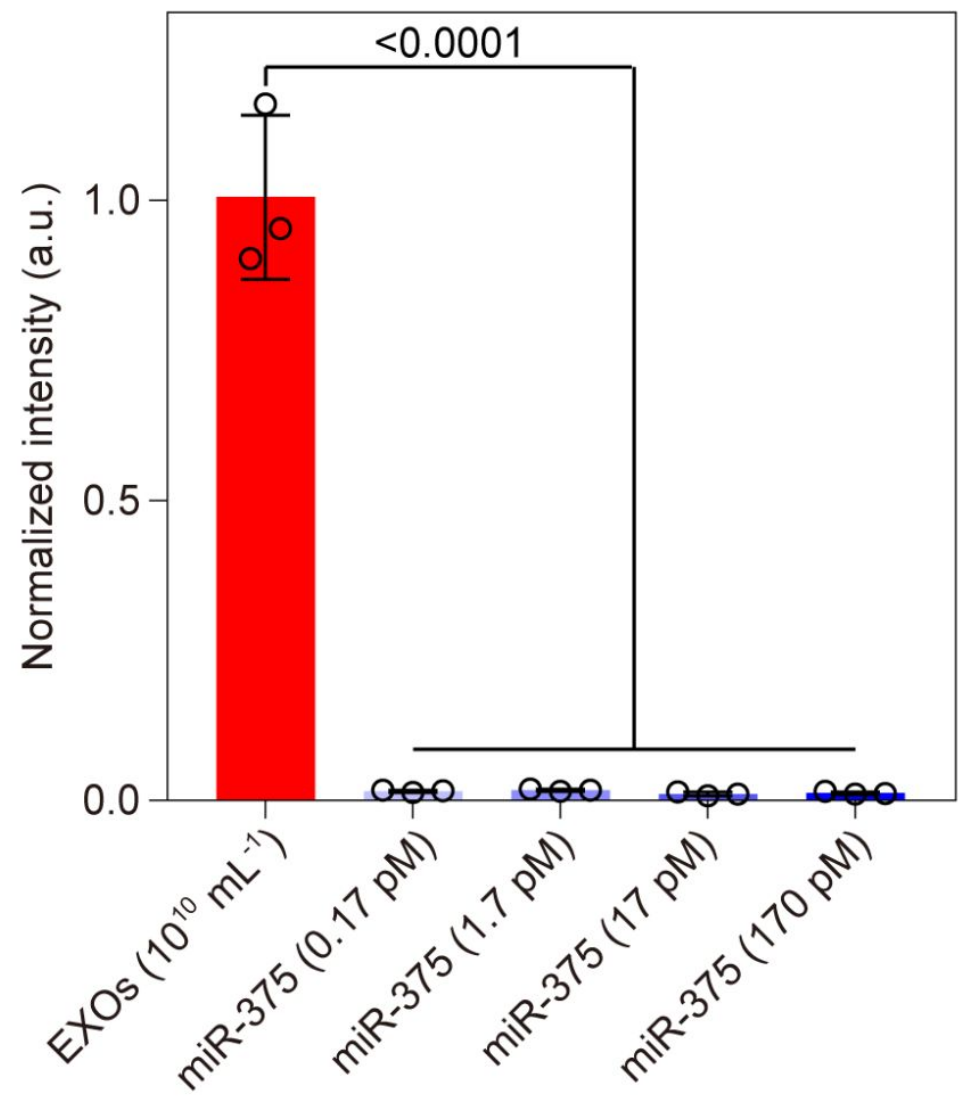

Figure S13. Weak fluorescence signal of synthetic miR-375 in TSN assay. $10^{10} \mathrm{~mL}^{-1}$ MCF-7 EXOs that containing 0.17 pM miR-375 and synthetic miR-375 with different concentrations ranging from $0.17 \mathrm{pM}$ to $170 \mathrm{pM}$ were analyzed by TSN $(n=3$, mean \pm s.d.). 


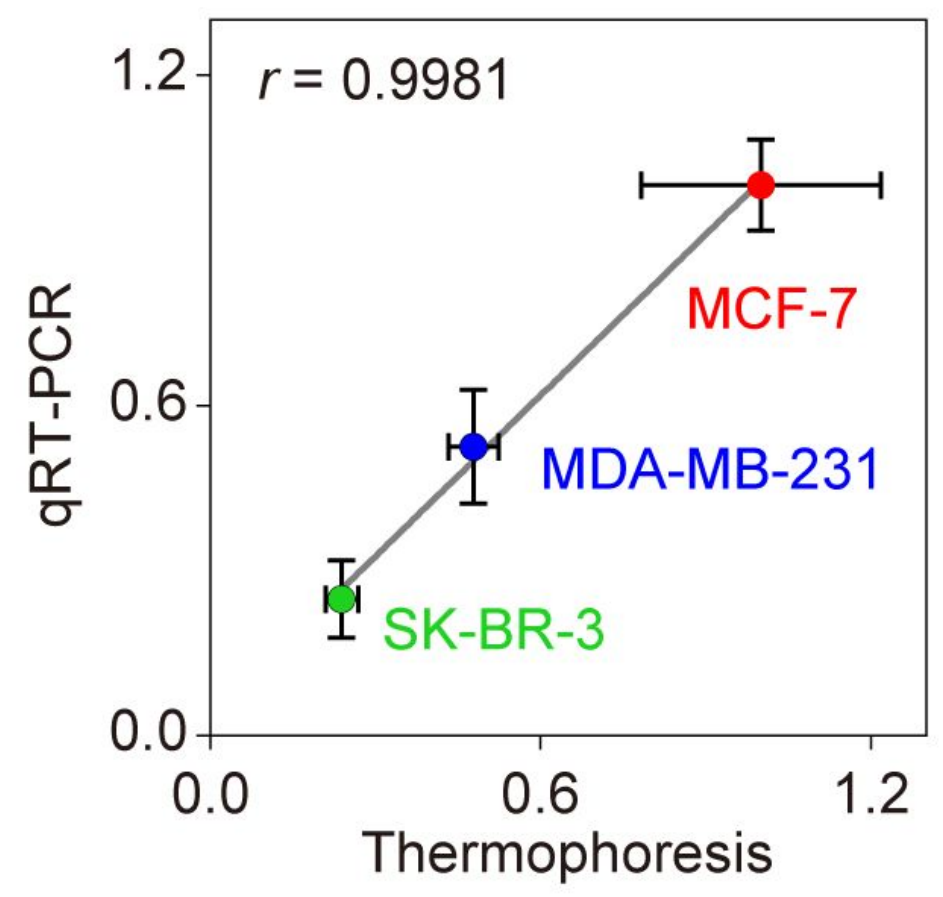

Figure S14. Correlation in the measured expression levels of exosomal miR-375 from different breast cancer cell lines between TSN and qRT-PCR $(n=3$, mean \pm s.d.). Spearman correlation coefficient $r$ was used to quantify the correlation. 


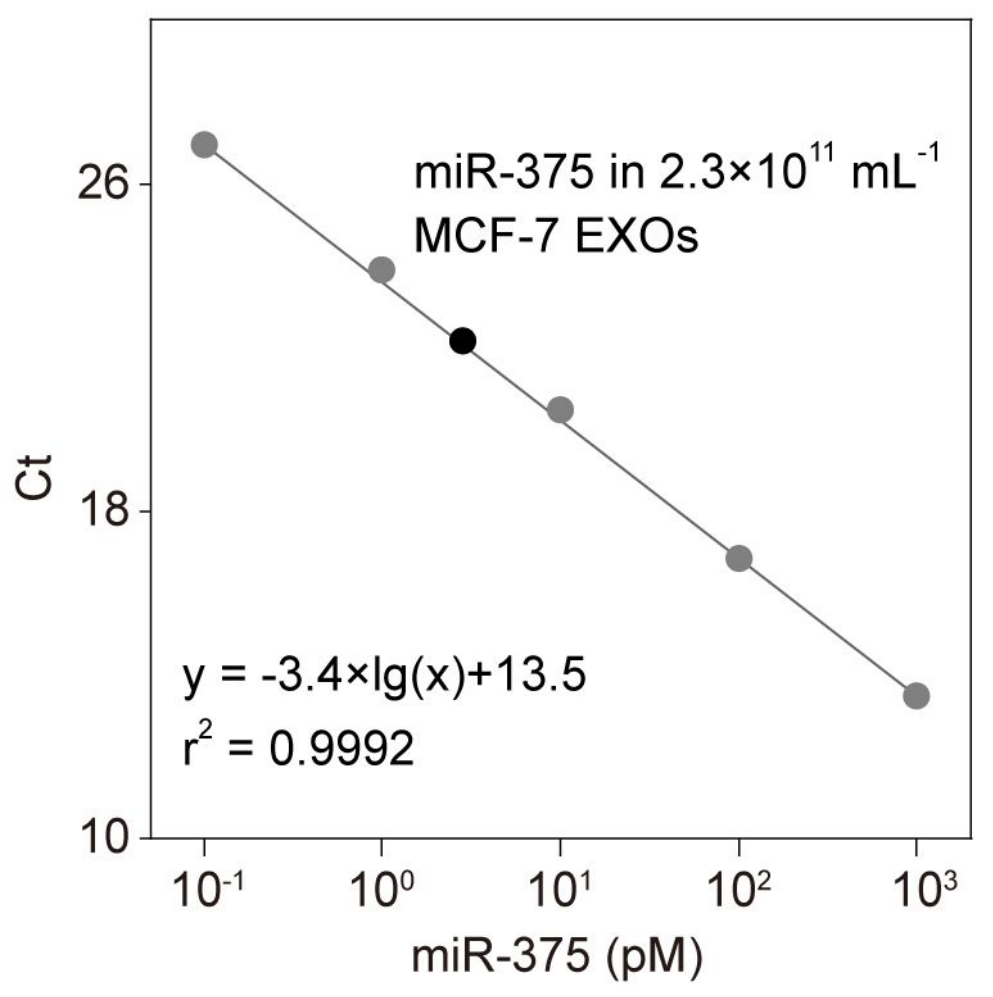

Figure S15. Standard curve for determining the absolute copy number of miR-375 in each MCF-7 EXO by qRT-PCR. 


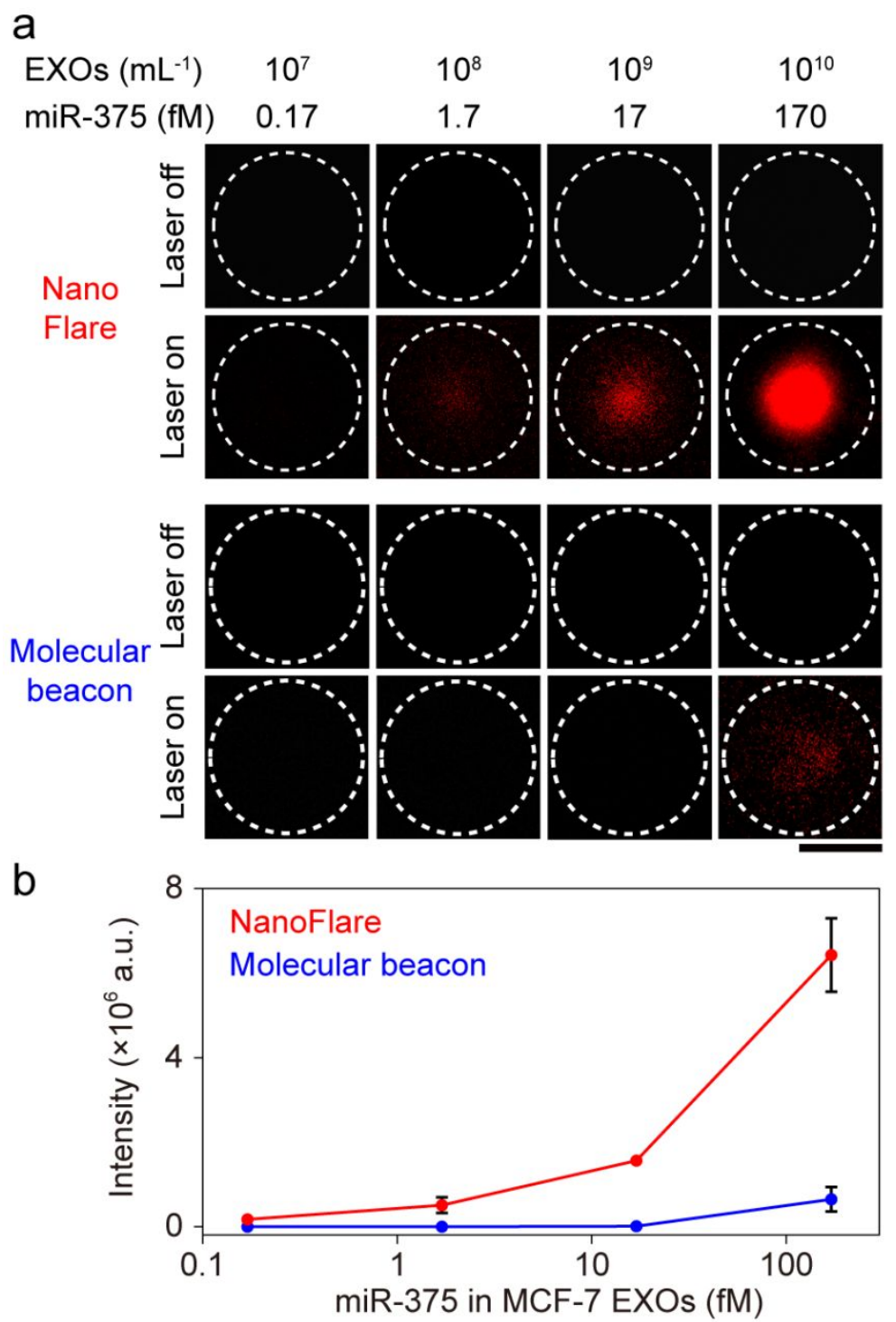

Figure S16. Comparative evaluation of NanoFlares and molecular beacons (MBs) for detecting miR-375 in MCF-7 EXOs. (a) Fluorescence images and (b) intensities of NanoFlare-based and Lipo/MB complex-based thermophoretic detection of miR-375 in MCF-7 EXOs prepared at different concentrations of $10^{7}-10^{10} \mathrm{~mL}^{-1}(n=3$, mean \pm s.d.). EXOs were incubated with $0.2 \mathrm{nM}$ NanoFlares (miR) or $9 \mathrm{nM} \mathrm{MBs}$ in Lipo/MB complexes, and subjected to 10 min laser heating. Scale bar, $50 \mu \mathrm{m}$. 


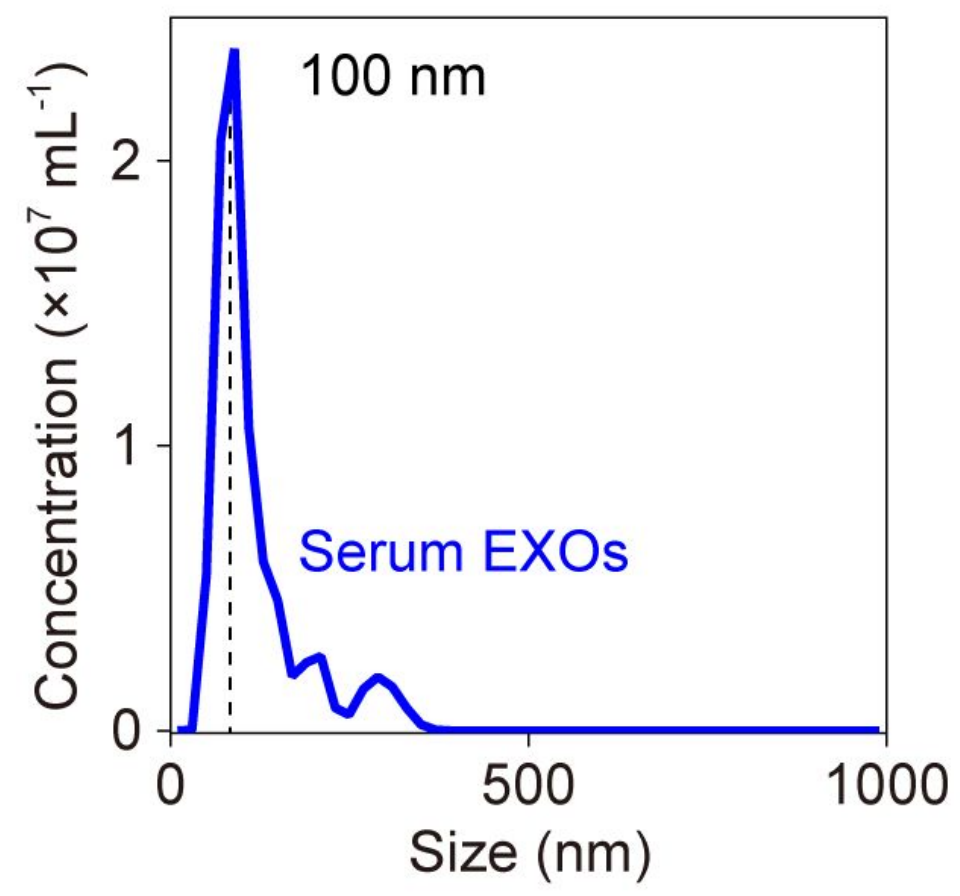

Figure S17. NTA measurement of the size distribution of serum EXOs from a given breast cancer patient. 
a

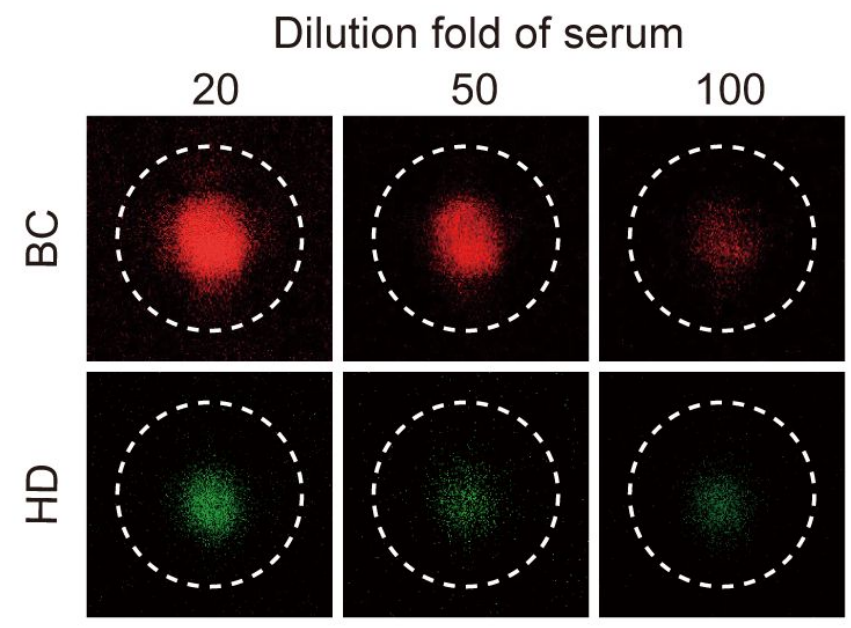

b

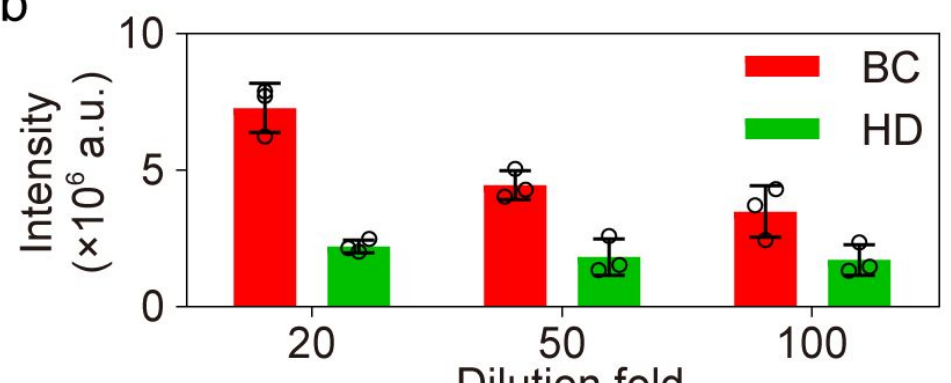

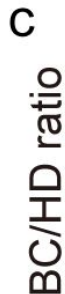

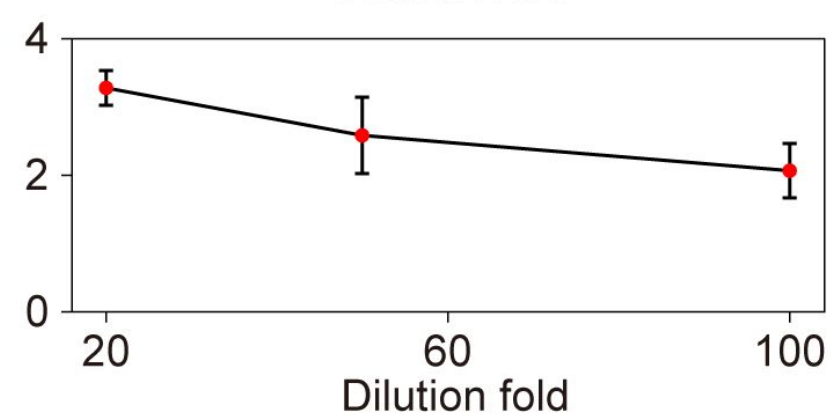

Figure S18. Optimization of dilution folds of serum. (a) Fluorescence images and (b) fluorescence intensities for TSN analyses of serum EXOs from a breast cancer patient (BC) and a healthy donor (HD) at different serum dilution folds (20 to 100). (c) Intensity ratio of $\mathrm{BC}$ to $\mathrm{HD}$ achieved the maximum at serum dilution fold of $20(n=$ 3 , mean \pm s.d.). 


\section{References}

S1 Fang, X.; Liu, X.; Schuster, S.; Tan, W., Designing a Novel Molecular Beacon for Surface-Immobilized DNA Hybridization Studies. J. Am. Chem. Soc. 1999, 121, 2921-2922.

S2 Ojea-Jiménez, I.; Bastús, N. G.; Puntes, V., Influence of the Sequence of the Reagents Addition in the Citrate-Mediated Synthesis of Gold Nanoparticles. J. Phys. Chem. C 2011, 115, 15752-15757.

S3 Liu, B.; Liu, J., Freezing Directed Construction of Bio/Nano Interfaces: Reagentless Conjugation, Denser Spherical Nucleic Acids, and Better Nanoflares. $J$. Am. Chem. Soc. 2017, 139, 9471-9474.

S4 Song, Y.; Shi, Y.; Huang, M.; Wang, W.; Wang, Y.; Cheng, J.; Lei, Z.; Zhu, Z.; Yang, C. J., Bioinspired Engineering of Multivalent Aptamer-Functionalized Nanointerface to Enhance Capture and Release of Circulating Tumor Cells. Angew. Chem. Int. Ed. 2019, 58, 2236-2240.

S5 Seferos, D. S.; Giljohann, D. A.; Hill, H. D.; Prigodich, A. E.; Mirkin, C. A., Nano-Flares: Probes for Transfection and mRNA Detection in Living Cells. J. Am. Chem. Soc. 2007, 129, 15477-15479.

S6 Prigodich, A. E.; Randeria, P. S.; Briley, W. E.; Kim, N. J.; Daniel, W. L.; Giljohann, D. A.; Mirkin, C. A., Multiplexed Nanoflares: mRNA Detection in Live Cells. Anal. Chem. 2012, 84, 2062-2066.

S7 Chevillet, J. R.; Kang, Q.; Ruf, I. K.; Briggs, H. A.; Vojtech, L. N.; Hughes, S.

M.; Cheng, H. H.; Arroyo, J. D.; Meredith, E. K.; Gallichotte, E. N.; 
Pogosova-Agadjanyan, E. L.; Morrissey, C.; Stirewalt, D. L.; Hladik, F.; Yu, E. Y.; Higano, C. S.; Tewari, M., Quantitative and stoichiometric analysis of the microRNA content of exosomes. Proc. Natl. Acad. Sci. U. S. A. 2014, 111, 14888-14893.

S8 Lee, J. H.; Kim, J. A.; Jeong, S.; Rhee, W. J., Simultaneous and multiplexed detection of exosome microRNAs using molecular beacons. Biosens. Bioelectron. 2016, $86,202-210$. 